\title{
Editorial: Plastics, Microplastics, and Nanoplastics: Management and Mitigation of Water Contamination
}

\author{
Joginder Singh ${ }^{1 *}$, Jastin Samuel ${ }^{1,2}$ and Rachel Hurley ${ }^{3}$ \\ ${ }^{1}$ Department of Microbiology, Lovely Professional University, Phagwara, India, ${ }^{2}$ Waste Valorization Research Lab, Lovely \\ Professional University, Phagwara, India, ${ }^{3}$ Norwegian Institute for Water Research, Oslo, Norway
}

Keywords: microplastic, pollution, source, fate, treatment, nanoplastic

Editorial on the Research Topic

Plastics, Microplastics, and Nanoplastics: Management and Mitigation of Water Contamination

\section{INTRODUCTION}

The properties of plastic have established it as an essential material in modern society. Yet, the extensive use of plastic and, in particular, mismanaged disposal of plastic products have culminated in environmental pollution that now spans a global scale (Geyer et al., 2017; Rochman, 2020). This contamination exists across wide size spectrum of plastic, from larger plastic litter down to smaller particles known as micro- and nanoplastics. This issue has received substantial attention from scientists, governments, media and the public, but there are still several persistent knowledge gaps in our understanding of the sources and fate of plastics, the associated risks and potential solutions for reducing pollution and its impacts.

Papers in this Research Topic address several critical components of microplastic pollution. From sources to solutions, these contributions advance understanding of microplastic distributions in the environment, establish the potential risks of microplastics, and articulate effective and appropriate strategies to minimise pollution into the future.

*Correspondence:

Joginder Singh

joginder.15005@/pu.co.in

Specialty section:

This article was submitted to

Toxicology, Pollution and the

Environment,

a section of the journal

Frontiers in Environmental Science

Received: 28 October 2021

Accepted: 29 October 2021

Published: 19 November 2021

Citation:

Singh J, Samuel J and Hurley R (2021)

Editorial: Plastics, Microplastics, and

Nanoplastics: Management and

Mitigation of Water Contamination.

Front. Environ. Sci. 9:803551

doi: 10.3389/fenvs.2021.803551

\section{PLASTICS ENTERING THE ENVIRONMENT: SOURCES AND ASSEMBLAGES}

Jemaa et al. and Herzke et al. addressed the sources of plastic to two contrasting environments: the Lebanese coastal environment and an Arctic fjord, respectively. In the former, Jemaa et al. attributed high concentrations observed in proximity to the Dora district of Beirut to the influence of the Beirut river inputting microplastics to the coastal environment, as well as additional contributions from the economic harbour of Beirut and the presence of a coastal landfill site. The highest concentrations were reported for the Manara region of Lebanon, where the sampling site is impacted by the outflow of wastewater effluent. This highlights anthropogenic pressures and proximity to different waste handling systems as an important source of (micro)plastics in this region. Numerically, plastics-and, in particular, microplastics-constituted the majority of debris collected in the sampling. This shines a light on the dominance of plastic in what is released from different waste handling sources.

The importance of wastewater systems is also highlighted by Herzke et al., who examined the release of microfibres from wastewater effluent into Adventfjorden close to Longyearbyen, the capital 
of the Arctic Svalbard archipelago. Here, the regional context is in stark contrast to the densely populated regions along the Lebanese coast. Yet, the retention capacity of wastewater treatment systems was highlighted as an important additional dimension to consider when evaluating the potential magnitude of this environmental release pathway in acting as a source of small plastic particles to the environment. High loads of microfibres were calculated to be released due to the limited degree of treatment, which represents an important component when assessing wastewater systems as a potential source of plastic contamination to the environment.

The diversity of plastic pollution released to the environment should not be overlooked. A high degree of heterogeneity has been reported globally in the plastic assemblages observed in different environmental samples, with regard to characteristics such as particle morphology, particle size, polymer type, and type and extent of particle aging or degradation. Nomenclature for plastic pollution-macroplastic, microplastic, nanoplastic, etc.-represent umbrella terms encompassing a wide range of different items with an array of different characteristics. This diversity was also featured in Jemaa et al., who report a range of different particle types, colour, and polymeric composition observed for both macro- and microplastic.

\section{FOLLOWING THE FATE AND TRANSPORT OF PLASTIC IN THE ENVIRONMENT}

This diversity in plastic pollution is also significant for the behaviour and ultimate fate of a given particle once inputted into the environment. Herzke et al. highlighted the importance of density on the fate and transport of microfibres in an Arctic fjord environment. Low density fibres are more easily entrained in surface waters and are rapidly exported from the fjord by the outgoing currents. In contrast, the densest fibres quickly sink and are deposited in the bottom sediments of the fjord. This creates a spatial pattern of accumulation zones, which are influenced by both proximity to sources and the net result of environmental processes governing fate and transport. The processes which continue to redistribute fibres across long timescales are still poorly understood, including the thresholds for currents to remobilise fibres from the fjord bed or the spatiotemporal variability of flow dynamics in the fjord environment.

Some spatial patterns in microplastic concentrations were reported by Jemaa et al, but these were not found to be significant. Instead, seasonality imparted a control on the temporal pattern of microplastic distributions. Higher concentrations were reported during spring sampling, which corresponds with the wet season in the Lebanon region. Rainfall can increase the connectivity between terrestrial and freshwater systems and surface runoff can transport particles into and through rivers, flushing them from catchments. Further research is needed to better understand the fate and transport of microplastics in a range of environment, to facilitate improved interpretation of observed microplastic distributions from monitoring activities.

\section{UNDERSTANDING POTENTIAL ENVIRONMENTAL RISKS OF PLASTIC POLLUTION}

One of the main drivers behind the rapid increase in attention paid to plastic pollution globally is the potential for such pollution to cause harm. This is particularly relevant for small particles, such as micro- and nanoplastics. As of yet, this harm is not welldefined, which relates back to the high degree of diversity in microplastic assemblages, as well as several persistent knowledge gaps linked to the lack of validated analytical methods for analysing nanoplastic in a range of environmental samples. The diversity imparts an important control on the potential for a particle to generate negative impacts on a given organism, for example physical effects related to particle size or shape. One mechanism through which micro- and nanoplastics could present a hazard is in their role as a vector for other, toxic contaminants. Agboola and Benson reviewed this in the context of organic contaminants, investigating the sorption dynamics between different plastics and different persistent organic pollutants (POPs). Still much work is needed to better understand the linkages between plastics and organic contaminants, but it was possible to establish that the hydrophobicity of the POPs and the surface area-to-volume ratio and polymer composition of the particle represent significant factors. In particular, polyethylene was identified as having the highest sorption capacity, which is significant given the abundance of this polymer type in environmental assemblages of microplastic, including in the work of Jemaa et al.

The linkages and sorption dynamics related to organic contaminants and microplastics thus represents a potential hazard associated with micro- and nanoplastic pollution. Yet, further research is urgently required to elucidate the relationship and potential risks. Agboola and Benson highlighted several important factors from their review, including: 1) more research using natural aged particles; 2) more research on the fate and bioavailability of micro- and nanoplastics and sorbed contaminants; and 3) more studies that incorporate a higher degree of environmental realism to better represent relevant environmental scenarios. This work is needed to underpin a more complete and holistic understanding of the potential risks posed by small plastic particles in the environment.

Risk is the net result of two components: hazard and exposure. Exposure in this context refers to the coexistence of microplastics and species that may be harmed by microplastic in the same space. This spatial component of risk was examined by Herzke et al., who projected their modelled microfibre concentrations over distributions of marine organisms in the study area. Benthic species were found to have the greatest potential exposure to fibres from wastewater in Adventfjorden. Denser polymer types, or particles that have more extensive biofouling, represent the particles that coalesce in the benthic zone, highlighting some particle characteristics which could be considered when establishing experiments to test potential hazards. Herzke et al. also considered seasonality in benthic community size and composition, which will alter exposure dynamics. This was also a component of the work of Jemaa et al., who conducted 
a preliminary risk assessment and observed that seasonal patterns were more important that spatiality in the context of risk. The highlights the needs to consider a temporal, as well as spatial, dimension when assessing the potential risks of microplastics in the environment.

\section{TOWARDS SOLUTIONS FOR PLASTIC POLLUTIONS}

Global plastic pollution has received considerable attention and this attention has in turninitiated important dialogue and generated innovation towards mitigation and remediation action to solve the pollution problem. One solution that has been postulated is the use of biodegradable plastics as an alternative material for many applications of plastic. Catarci Carteny and Blust present an important perspective on the environmental reality of this option: materials certified under current standards for biodegradability are not necessarily biodegradable in the environment that they end up in. Their work investigates the degradation of two biodegradable and one conventional polymer in a simulated marine environment and find that, over the timescales set in current biodegradation standards, no consistent degradation was observed, and the biodegradable polymers did not stand out compared to polyethylene, the conventional polymer used in the study. This calls into question the realistic applicability of current standards to establish an alternative material used in real scenarios which is effective in reducing pollution. In many cases, biodegradable polymers may not necessarily represent a viable solution. This highlights the need to test specific materials in specific situations, which resemble realistic scenarios of environmental conditions and the fate of plastic pollution. Communication and dissemination of scientific evidence is also addressed, including the need for better labelling and awareness amongst consumers related to the true value of biodegradable polymers in some applications.

An alternative solution is presented by Adelodun. This review presents the viability of energy recovery through optimized pyrolysis in reducing plastic pollution. Plastic waste presents an opportunity for producing profit, through the generation of high calorific fuel resulting from the pyrolysis process. This may

\section{REFERENCES}

Geyer, R., Jambeck, J. R., and Law, K. L. (2017). Production, Use, and Fate of All Plastics Ever Made. Sci. Adv. 3 (7), e1700782. doi:10.1126/ sciadv. 1700782

Rochman, C. M. (2018). Microplastics Research-From Sink to Source. Science 360 (6384), 28-29. doi:10.1126/science.aar7734

Conflict of Interest: The authors declare that the research was conducted in the absence of any commercial or financial relationships that could be construed as a potential conflict of interest. actually comprehensively exceed the value that can be obtained from other waste streams that are also presently subject to pyrolysis, such as paper or organic waste. This is based on the energy that is recoverable from the plastics and the potential added benefit from the use of catalysts to increase the efficiency of the recovery process (Adelodun). This can be used in combination with strategies to prioritise certain polymer types for use in particular products, based on their recyclability or potential for energy recovery, and encouraging better waste management and public awareness of plastic waste issues, for example to increase the efficacy of source separation of plastic.

This Research Topic spans many different important aspects inherent in the field of plastic pollution research. The diversity, and in particular multidisciplinarity, of articles reflects the wide array of different challenges that must still be solved to reduce plastic pollution in the environment. These contributions advance knowledge in the field and present critical agendas for future research, which sets a path for solving plastic pollution.

\section{AUTHOR CONTRIBUTIONS}

All authors listed have made a substantial, direct, and intellectual contribution to the work and approved it for publication.

\section{FUNDING}

The contribution of $\mathrm{RH}$ was supported under the scope of the India-Norway cooperation project INOPOL. RH gratefully acknowledges funding from the Norwegian Ministry of Foreign Affairs and the Royal Norwegian Embassy in New Delhi. The views expressed here are solely of the authors and not the funding organisation.

\section{ACKNOWLEDGMENTS}

The authors acknowledge the support of the Frontiers in Environmental Science Editorial Office, including the journal specialist Andrea Lazen who delivered important assistance in establishing and running this Research Topic.

Publisher's Note: All claims expressed in this article are solely those of the authors and do not necessarily represent those of their affiliated organizations, or those of the publisher, the editors and the reviewers. Any product that may be evaluated in this article, or claim that may be made by its manufacturer, is not guaranteed or endorsed by the publisher.

Copyright (C) 2021 Singh, Samuel and Hurley. This is an open-access article distributed under the terms of the Creative Commons Attribution License (CC BY). The use, distribution or reproduction in other forums is permitted, provided the original author(s) and the copyright owner(s) are credited and that the original publication in this journal is cited, in accordance with accepted academic practice. No use, distribution or reproduction is permitted which does not comply with these terms. 